INTERNATIONAL JOURNAL OF RESEARCHES IN BIOSCIENCES, AGRICULTURE AND TECHNOLOGY (c) VISHWASHANTI MULTIPURPOSE SOCIETY (Global Peace Multipurpose Society) R. No. MH-659/13(N) www.vmsindia.org

\title{
SYNTHESIS AND BIOLOGICAL ACTIVITY OF 2- SUBSTITUTED THIOHYDANTOIN
}

\author{
Sanjay V. Kolhe \\ P. G. Department of chemistry \\ Shri Shivaji Arts', Commerce \& Science College, Akot Dist. Akola \\ Email; -drsanjaykolhe22@gmail.com
}

\begin{abstract}
:
2 hydroxy substituted calcone (I a-d) was dissolved in DMSO and mercuric acetate was added to it. The reaction mixture was refluxed for 2-3 hours and then diluted with water. The solid separated was crystallized from rectified sprit to give coumarone 3-one i.e. aurone (II a-d). Substituted aurone further refluxed with thiourea in ethanolic $\mathrm{KOH}$ for 3 hours gives substituted thiohydantoin. The structural elucidation of compound was done on the basis of analytical and spectral data.
\end{abstract}

Key words: Synthesis, coumarone 3-one, mercuric acetate, urea, ethanolic $\mathrm{KOH}$, antimicrobial activity.

\section{INTRODUCTION:}

Thiohydantoins are sulfur analogs of hydantoins with one or both carbonyl groups replaced by thiocarbonyl groups ${ }^{1}$. Among the known thiohydantoins, 2-thiohydantoins are most notably known due of their wide applications as hypolipidemic ${ }^{2}$, anticarcinogenic ${ }^{3}$ , antimutagenic ${ }^{4}$, antithyroidal ${ }^{5}$, antiviral (e.g., against herpes simplex virus, HSV) ${ }^{6}$, human immunodeficiency virus (HIV) ${ }^{7}$ and tuberculosis ${ }^{8}$ , antimicrobial (antifungal and antibacterial) ${ }^{9}$, anti-ulcer and anti-inflammatory agents ${ }^{10}$, as well as pesticides ${ }^{11}$. Additionally, 2-thiohydantoins have been used as reference standards for the development of C-terminal protein sequencing ${ }^{12}$, as reagents for the development of dyes ${ }^{13}$ and in textile printing, metal cation complexation and polymerization catalysis ${ }^{14}$. It is therefore not surprising that various different synthetic methods have been developed to prepare 2thiohydantoin and its derivatives. Some of the most commonly used methods are the treatment of a-amino acids with acetic anhydride followed by ammonium thiocyanate ${ }^{15}$ and the coupling reaction between a-amino acid derivatives and isothiocyanate $4 \mathrm{a}, 12 \mathrm{~b}, 16$. Other preparative methods for 2- thiohydantoins include the reactions between thiourea and benzil17 thiourea and a-halo acids ${ }^{18}$, oxazolinone and thiocyanate ${ }^{19}$, amino amide and diimidazole thiocarbonate 20 , and others ${ }^{21}$.In addition, some of the above reactions have been modified to take place under microwave irradiation ${ }^{17 c}$ and solid-phase ${ }^{16 a, 22}$ or fluorous-phase 23 supported reaction conditions. However, the above methods often suffer from one or more synthetic limitations for large-scale preparation of 2-thiohydantoin derivatives due to their use of expensive, moisture sensitive and/or highly toxic starting materials and reagents. Moreover, the methods developed for combinatorial synthesis and used to prepare 2 - thiohydantoin derivatives in small quantities for purposes like biological testing may not be feasible when operated on a large scale $22 \mathrm{~d}, 24$ A Thiohydantoin derivative has also been reported as herbicidal ${ }^{25}$. Bucherer reaction has also been reported for the synthesis of thiohydantoin ${ }^{26}$. Sulfenylated thiohydantoins has also been reported as fungicides. Ant diabetic hydantoins have been synthesized by Japanese scientists ${ }^{27}$. 1-3-diglycidyl-5, 5-dimethyl hydantoin has been used for primed steel plate to give a good coating for weathering, alkali, acid and water resistance 28 . Some thiohydantoin derivatives have been used in the treatment of blood circulation disorder29. Some thiohydantoins have been reported as inhibitors of pyrimidine biosynthesis ${ }^{30}, 5$, 5-disubstituted thiohydantoins have also been synthesized for their anti HIV activity $^{31}$. Synthesis of benzylidene derivatives of 3(2,3,4-chlorophenyl) thiohydantoins are reported for their anticonvulsant properties ${ }^{32}$, 1-bromo thio-hydantoins is reported where transposition of halogen atom from nitrogen to 3-alkyl group is studied $^{33}$. 1-N-phenyl substituted 2thiohydantoins derivatives were synthesized by $Z$. Jinpei et al for their antinociceptive activity 34 . Acetylation of 3-substituted 1-aminothiohydantoins has been reported ${ }^{35}$. Reaction of 5-arylidene-3-phenyl-2-thiohydantoin with 2,3,4,6-tetra-o-acetyl-a-D-gluco-pyranosyl bromide are reported. The product is arylidenephenyl [(tetra acetyl glyco pyranosyl) thiohydantoin. 36 We now report a simple method for the preparation of 2-thiohydantoin derivatives that can easily be scaled up in the laboratory.

\section{EXPERIMENTAL:}

The melting points were taken in a capillary tube; IR spectra were recorded in Nijol, $1 \mathrm{H}$ NMR spectra were recorded in $\mathrm{CDCl}_{3}$ with TMS as an internal standard. The purity of 
A synthesized compound was check by TLC. The structural elucidation of compound was done on the basis of chemical and spectral data. The starting ketones were 2-hydroxy-5-bromo acetophenone (II a), 2-hydroxy-3-nitro-5-bromo acetophenone (II b). 2-Hydroxy-5-bromo acetophenone (II a) was prepared from para bromo phenyl acetate (I a) by Fries migration using anhydrous $\mathrm{AlCl}_{3}$. 2-Hydroxy-3-nitro-5bromo acetophenone (II b) was prepared by nitration of 2-hydroxy-5-bromo acetophenone (II a).

Synthesized substituted acetophenone (II a\&b) were condensed with substituted aldehyde separately to get corresponding chalcones (III ad).

The above synthesized substituted chalcones (III a-d) were refluxed in DMSO medium in presence of mercuric acetate catalyst to yield 2(substituted benzylidene)-7-substituted-5-bromo coumaran-3-one (IV a-d).

2-(substituted benzylidene)-7-substituted-5bromo coumaran-3-one (IV a-d) was refluxed with thiourea in presence of alkaline medium and alcohol to get 2-substituted thiohydantoin (V a-d).

Preparation of 5-(2-hydroxy-3-nitro-5-chloro phenyl) 5-(à-hydroxy-4-methoxy benzyl)-2-thiohydantion (II a)

2-(4' methoxy benzylidene)-5- bromo-7-nitro coumaran-3-one (I a) (0.01 mole) and

thiourea ( 0.01 mole) were dissolved in $40 \mathrm{ml}$ of ethanol. To this mixture $10 \mathrm{ml}$ of $10 \% \mathrm{KOH}$

was added drop wise with constant stirring, allowed to stand for 2 to 3 hours. The reaction mixture was refluxed for $3 \mathrm{hrs}$. Cooled and then diluted with ice cold water washed several time with $1 \% \mathrm{NaHCO}_{3}$ solution and then with distilled water. It was then crystallized from ethanol to get 5-(2-hydroxy-3-nitro-5-chloro phenyl) 5-(a-hydroxy-4-methoxy benzyl)-2-thio hydantion (II a).

The structure of compound (II a) has been supported by chemical and spectral data.

\section{Properties of the compound (II a)}

- Deep brown color crystalline solid m.p. $128^{\circ} \mathrm{C}$.

- It shows positive ferric chloride indicating noninvolvement of phenolic -OH group.

- An IR spectrum was recorded in Nijol.

I. $3852(-\mathrm{N}-\mathrm{H}$, stretching).

II. $3853(-\mathrm{N}-\mathrm{H}$, stretching).

III. 3815-3801 (-OH group stretching).

IV. 1875 (Lactum cyclic C=Sgroup stretching).

V. 1511 (-NO2 group symmetrical aromatic stretching).

VI. 1340 (-NO2 group unsymmetrical aromatic stretching).

VII 1251 (-NH bond stretching)

VIII. 1060 (-CHOH group stretching).

IX. $1480 \mathrm{~cm}-1$ (C-Br group stretching).

- $1 \mathrm{H}$ NMR in $\mathrm{CDCl} 3$ with TMS as an internal standard.

I. $1.25(\mathrm{~s}, 1 \mathrm{H},-\mathrm{CH})$.

II. 3.9 (s, 3H, Ar-OCH3 group).

III. 6.3-6.4 (broad, $1 \mathrm{H}-\mathrm{OH}$ ).

IV. $6.8(\mathrm{~m}, 6 \mathrm{H}, \mathrm{Ar}-\mathrm{H})$.

V. 6.9-7.8ठ (s, 1H, Ar-OH).

These chemical and spectral data shows that compound (II a) is got 5-(2-hydroxy-3- nitro -5-bromo phenyl) 5- (á-hydroxy-4-methoxy benzyl)-2-thiohydantoin. Similarly other compounds (II b-II d) were prepared by above method.<smiles>[R]c1ccc(C2(O)C(=O)NC(=S)NC2c2cc(Br)cc([R])c2O)c([R])c1[R]</smiles>

II a-d

III a-d

Table 1: Synthesized compounds, M.P.'s and yields

\begin{tabular}{|c|c|l|l|l|l|c|c|}
\hline $\begin{array}{l}\text { S. } \\
\text { No. }\end{array}$ & Compounds & $\mathrm{R}_{1}$ & $\mathrm{R}_{2}$ & $\mathrm{R}_{3}$ & $\mathrm{R}_{4}$ & M. P. $\left({ }^{\circ} \mathrm{C}\right)$ & Yield (\%) \\
\hline 1 & $\mathrm{II} \mathrm{a}$ & $\mathrm{NO}_{2}$ & $\mathrm{OCH}_{3}$ & $\mathrm{H}$ & $\mathrm{H}$ & $126-128$ & 76 \\
\hline 2 & $\mathrm{II} \mathrm{b}$ & $\mathrm{NO}_{2}$ & $\mathrm{H}$ & $\mathrm{H}$ & $\mathrm{OH}$ & $124-1130$ & 58 \\
\hline 3 & $\mathrm{II} \mathrm{c}$ & $\mathrm{H}$ & $\mathrm{H}$ & $\mathrm{H}$ & $\mathrm{H}$ & $143-148$ & 61 \\
\hline 4 & $\mathrm{II} \mathrm{d}$ & $\mathrm{H}$ & $\mathrm{H}$ & $\mathrm{NO}_{2}$ & $\mathrm{H}$ & $156-160$ & 71 \\
\hline
\end{tabular}


Biological assay of the synthesized products:

The antimicrobial effects of thiohydantoin against clinically isolated microbes such as Escherichia coli, Staphylococcus aureus, Klebsilla, Pseudomons were studied. The technique used for this investigation was a diskdiffusion method on a nutrient agar medium. Sterilized nutrient agar was poured into Petri dish and allowed it to solidify. After solidification of the medium, a bacterial culture was spread over it uniformly with spreader. After it, a sterilized paper disc of size $4 \mathrm{~mm}$ immersed in sample was inoculated on the medium with bacterial culture. This was done separately for different bacterial cultures. The plates were incubated overnight at $37^{\circ} \mathrm{C}$ to check the bacterial growth and drugs sensitivity (zone of inhibition). The area around disks that has no significant growth is referred to as the zone of inhibition. The zone of inhibition was observed on next day for different bacterial culture which was measured in $\mathrm{mm}$. This experimental data was represented in the following table.

Table 2: Antibacterial activity data of the heterocyclic derivatives of aurones

\begin{tabular}{|l|l|l|l|l|}
\hline Compound & E. coli & S. aureus & Klebsiella & Pseudomons \\
\hline IIa & $20.3 \mathrm{~mm}$ & $12.7 \mathrm{~mm}$ & $15.1 \mathrm{~mm}$ & $18.2 \mathrm{~mm}$ \\
\hline IIb & $22.0 \mathrm{~mm}$ & $14.8 \mathrm{~mm}$ & $14.4 \mathrm{~mm}$ & $14.0 \mathrm{~mm}$ \\
\hline IIC & $19.5 \mathrm{~mm}$ & $20.0 \mathrm{~mm}$ & $17.3 \mathrm{~mm}$ & $18.8 \mathrm{~mm}$ \\
\hline IId & $18.3 \mathrm{~mm}$ & $12.2 \mathrm{~mm}$ & $16.0 \mathrm{~mm}$ & $19.7 \mathrm{~mm}$ \\
\hline
\end{tabular}

The compound IIb showed maximum inhibitory activity of $22.0 \mathrm{~mm}$ one of inhibition for $E$. coli . Compounds IIb showed maximum inhibitory activity of $21.3 \mathrm{~mm}$ for Klebsilla. and Compound IId showed maximum inhibitory activity of $19.7 \mathrm{~mm}$ for Pseudomons and IIc showed maximum inhibitory activity of 19.5 and $20.0 \mathrm{~mm}$ for S. aureus; E.coli

The preliminary results of our study showed that substituted 2- thiohydantoin could serve as potential drug for preparation of pesticide and disinfectant. Further investigation are needed to anticonvulsants in the treatment of seizer disorder.
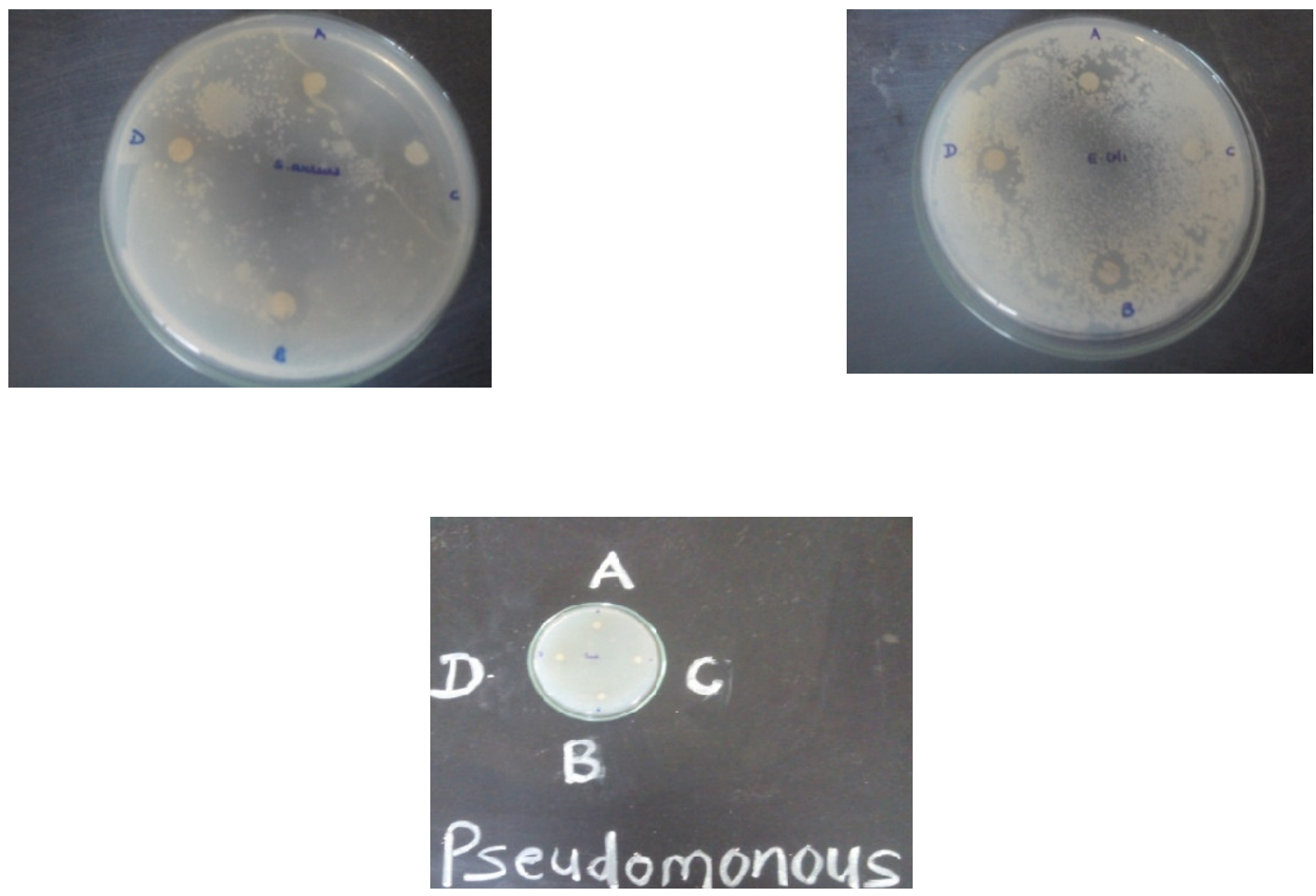


\section{REFERENCES:}

1. Johnson, T. B.; Chernoff, L. H. J. Am. Chem. Soc. 1913, 35, 1208.

2. . (a) Tompkins, J. E. J. Med. Chem. 1986, 29, 855; (b) Elwood, J. C.; Richert, D. A.; Westerfeld, W.W. Biochem. Pharmacol. 1972, 21,1127

3. Al-Obaid, A. M.; El-Subbagh, H. I.; Khodair, A. I.; Elmazar, M. M. Anticancer Drugs 1996, 7, 873.

4. (a) Takahashi, A.; Matsuoka, H.; Ozawa, Y.; Uda, Y. J. Agric. Food Chem. 1998, 46, 5037; (b) Froelich, E.; Fruehan, A.; Jackman, M.; Kirchner, F. K.; Alexander, E. J.; Archer, S. J. Am. Chem. Soc. 1954, 76, 3099.

5. (a) Marx, J. V.; Richert, D. A.; Westerfeld, W. W. J. Med. Chem. 1970, 13, 1179; (b)

6. Cheymol, J.; Chabrier, P.; Gay, Y.; Lavedan, J. P. Arch. Int. Pharmacodyn. Ther. 1951, 88, 342; (c) Cheymol, J.; Chabrier, P.; Gay, Y. Arch. Int. Pharmacodyn. Ther. 1951, 87, 321.

7. El-Barbary, A. A.; Khodair, A. I.; Pedersen, E. B.; Nielsen, C. J. Med. Chem. 1994, 37, 73.

8. (a) Chérouvrier, J.-R.; Carreaux, F.; Bazureau, J. P. Molecules, 2004, 9, 867, and references citedntherein; (b) Khodair, A. I.; El-Subbagh, H. I.; El-Emam, A. A. Boll. Chim. Farm. 1997, 136,561

9. Archer, S.; Unser, M. J.; Froelich, E. J. Am. Chem. Soc. 1956, 78, 6182.

10. (a) Lacroix, G.; Bascou, J.-P.; Perez, J.; Gadras, A. U. S. Pat. 6,018,052, 2000; (b) Lacroix, G.; Bascou, J.-P.; Perez, J.; Gadras, A. U. S. Pat. 5,650,519, 1997; (c) Marton, J.; Enisz, J.; Hosztafi,S.; Timar, T. J. Agric. Food Chem. 1993, 41, 148.

11. Curran, A. C. W. U. S. Pat. 3,984,430, 1976.

12. Nagpal, K. L. U. S. Pat. 4,473,393, 1984.

13. (a) Mo, B.; Li, J.; Liang, S. Anal. Biochem. 1997, 249, 207; (b) Cromwellt, L. D.; Stark, G. R. Biochemistry 1969, 8, 4735.

14. . (a) Nelson, J. V.; Helber, M. J.; Brick, M. C. U. S. Pat. 5,695,917, 1997; (b) Ooi, T.; Fukui, T.; Kobayashi, M.; Ueno, K.; Kagami, K.; Suzuki, M.; Nishino, K. U. S. Pat. 5,482,814, 1996.

15. Kandil, S. S.; El-Hefnawy, G. B.; Baker, E. A. Thermochim. Acta 2004, 414, 105.

16. (a) Wyzlic, I. M.; Tjarks, W.; Soloway, A. H.; Perkins, D. J.; Burgos, M.; O'Reilly, K. P. Inorg.Chem. 1996, 35, 4541; (b) Johnson, T. B.; Bengis, R. J. Am. Chem. Soc. 1913, 35, 1605; (c)Johnson, T. B.; Nicolet, B. H. J. Am. Chem. Soc. 1912, 34, 1973; (d) Johnson, T. B.; Scott, W. M. J. Am. Chem. Soc. 1913, 35, 1130 .

17. (a) Li, J.-P.; Ma, C.-M.; Qu, G.-R. Synth. Commun. 2005, 35, 1203; (b) Innocenti, A.; Casini, A.; Alcaro, M. C.; Papini, A. M.; Scozzafava, A.; Supuran, C. T. J. Med. Chem. 2004, 47, 5224; (c) Elokdah, H.; Sulkowsk, T. S.; Abou-Gharbia, M.; Butera, J. A.; Chai, S.Y.; McFarlane, G. R.; McKean, M. L.; Babiak, J. L.; Adelman, S. J.; Quinet, E. M. J. Med.
Chem. 2004, 47, 681; (d) Yeh, W.-B.; Lin, M.J.; Lee, M.-J.; Sun, C.-M. Mol. Divers. 2003, 7, 185; (e) Erve, J. C. L.; Amarnath, V.; Sills, R. C.; Morgan, D. L.; Valentine, W. M. Chem. Res. Toxicol. 1998, 11, 1128; (f) Marx, J. V.; Richert, D. A.; Westerfeld, W. W. J. Med. Chem. 1970, 13, 1179; (g) Johnson, T.B.; Hill, A. J.; Kelsey, E. B. J. Am. Chem. Soc. 1920, 42,1711

18. (a) Muccioli, G. G.; Martin, D.; Scriba, G. K. E.; Poppitz, W.; Poupaert, J. H.; Wouters, J.;Lambert, D. M. J. Med. Chem. 2005, 48, 2509; (b) Muccioli, G. G.; Poupaert, J. H.; Wouters, J.;Norberg, B.; Poppitz, W.; Scriba, G. K. E.; Lambert, D. M. Tetrahedron 2003, 59, 1301; (c)Muccioli, G. G.; Wouters, J.; Poupaert, J. H.; Norberg, B.; Poppitz, W.; Scriba, G. K. E.; Lambert,D. M. Org. Lett. 2003, 5, 3599.

19. Mahmoud, A. M.; Abdel-Rahman, A. E.; ElNaggar, G. M.; El-Sherief, H. A. Indian J. Chem.Sect. B 1984, 23B, 379.

20. Chain, E. B. The Chemical Structure of the Penicillins; Nobel Lecture, March 20, 1946. Available at http://nobelprize.org/nobel_prizes/medicine/1 aureates / 1945/chain-lecture.pdf.

21. . (a) Wang, X.-J.; Zhang, L.; Xu, Y. B.; Krishnamurthy, D.; Varsolona, R.; Nummy, L.; Shen, S.;Frutos, R. P.; Byrne, D.; Chung, J. C.; Farina, V.; Senanayake, C. H. Tetrahedron Lett. 2005, 46, 273; (b) Nefzi, A.; Giulianotti, M. A.; Houghten, R. A. Tetrahedron Lett. 2000, 41, 2283.

22. . (a) Zhang, W.; Lu, Y. Org. Lett. 2003, 5, 2555; (b) Zhang, W. Tetrahedron, 2003, 59, 4475; (c) Gasch, C.; Salameh, B. A. B.; Pradera, M. A.; Fuentos, J. Tetrahedron Lett. 2001, 42, 8615; (d)Somsák, L.; Nagy, V.; Docsa, T.; Tóth, B.; Gergely, P. Tetrahedron: Asymmetr. 2000, 11, 405; (e) Floch, L.; Oremus, V.; Kovac, M. Molecules 1999, 4, 279; (f) Morin, J. M.; Ternansky, R. J.; Noreen, R.; Lind, P. T. U. S. Pat. 5,714,503, 1998; (g) Boyd, V. L.; Bozzini, M.; DeFranco, R. J. U. S. Pat. 5,665,603, 1997; (h) Morin, J. M.; Ternansky, R. J.; Noreen, R.; Lind, P. T. U. S. Pat. 5,658,907, 1997; (i) Sim, M. M.; Ganesan, A. J. Org. Chem. 1997, 62, 3230. Molecules 2006, 11750

23. (a) Park, K. H.; Kurth, M. J. J. Org. Chem. 1999, 64, 9297; (b) Karnbrock, W.; Deeg, M.;Gerhardt, J.; Rapp, W. Mol. Divers. 1998, 4, 165; (c) Matthews, J.; Rivero, R. A. J. Org. Chem.1997, 62, 6090.

24. Kim, S. W.; Ahn, S. Y.; Koh, J. S.; Lee, J. H.; Ro, S.; Cho, H. Y. Tetrahedron Lett. 1997, 38, 4603.

25. Ganesan, A. Pure Appl. Chem. 2001, 73, 1033.

26. Schroder, Ludwing et al., Eur. Pat. Appl. Ep., 91, 596, 19, Oct. (1983), DE. Appl. 3, 213140, 08 Apr. (1982), pp.47.

27. Bowness Garry W., Balbir S. et al. J. Chem. Soc., Perkin, Trans-I, (ii) (1983), 2649- 53 (ENG.). 
28. Eisiac Co. Ltd. Jpn. Kokai Tokkyo Koho JP, 58, 213, 717 (83,213,717), (1983), Appl. 83/6085, 20 Jan 1982, 18.

29. Kurihara, Kenji, Kasahara et al.,Japan Kokai, $78,19,348$ (15336-81-9).

30. Kuron, Masatsune et al. Jpn. Kokai Tokkyo, Koho JP., 40,145,023, 05, (1990), 7.

3 1. Howie, Colin, 177 (1989), 189 (Eng.).

32. Comber, Robert N., Revnolds, J. Med. Chem., 35(19) (1992), Robert C., et al.3567-72 (Eng.).

33. Rydzik, Elfryda, Kaminoka Anna, Pol. Pharm., 41(4) (1984), 459-64 (Pol.).

34. Suarez A.R., Arguello B.V., An. Asoc. Quim Argent., 72(5) (1984),493-9 (Eng.).
35. Zhou, Zinpei et al., Zbongguo Yooke Dacue Auebao, 22(6) (1991), 330-3 (Ch.).

36. Ayupova A.T., Khim G.G., Geterotsiki, Soedin, (ii) (1991), 1512-14 (Russ.).

37. El-Barbary A.A., Saafan A.A. et al.Delta J. Sci., 14(2) (1990), 601-22 (Eng.).

38. Blanc M., Cussac M., Eur. J. Med. Chem., 27(3) (1992), Boucherie A., 267-75 (Fr.).

39. El-Barbary A.A., Saafan A.A. et al.Delta J. Sci., 14(2) (1990), 623-46 (Eng.).

40. Aboutabi M.A., Abdel Rehmoan, J. Chem. Technol. Biotechnol. Chem. Technol., 33A(6) (1983), 286-93 (Eng.). 